\title{
Design of a Selective and Sensitive PVC-Membrane Potentiometric Sensor for Strontium Ion Based on 1,10-Diaza-5,6-benzo-4,7-dioxacyclohexadecane-2,9-dione as a Neutral Ionophore
}

\author{
Mojtaba Shamsipur ${ }^{1, *}$, Sayed Yahya Kazemi ${ }^{2}$ and Hashem Sharghi $^{3}$ \\ 1 Department of Chemistry, Razi University, Kermanshah, Iran; E-mail: mshamsipur@ yahoo.com \\ 2 Department of Science, College of Agriculture, Mazandaran University, Sari, Iran \\ 3 Department of Chemistry, Shiraz University, Shiraz, Iran \\ * Author to whom correspondence should be addressed.
}

Received: 6 February 2007 / Accepted:16 March 2007 / Published: 12 April 2007

\begin{abstract}
A novel PVC membrane sensor for the $\mathrm{Sr}^{2+}$ ion based on 1,10-diaza-5,6-benzo-4,7dioxacyclohexadecane-2,9-dione has been prepared. The sensor possesses a Nernstian slope of $30.0 \pm 0.6 \mathrm{mV}$ decade $^{-1}$ over a wide linear concentration range of $1.6 \times 10^{-6}-3.0 \times 10^{-3} \mathrm{M}$ with a detection limit of $6.3 \times 10^{-7} \mathrm{M}$. It has a fast response time of $<15 \mathrm{~s}$ and can be used for at least two months without any considerable divergence in potential. The potentiometric response is independent of the $\mathrm{pH}$ of test solution in the $\mathrm{pH}$ range 4.3-9.4. The proposed electrode shows good selectivities over a variety of alkali, alkaline earth, and transition metal ions.
\end{abstract}

Keywords: ion-selective electrode, $\mathrm{Sr}^{2+}$, PVC-membrane, macrocyclic diamide, potentiometry

\section{Introduction}

Strontium is widely distributed in many biological systems and throughout the environment, at various concentration levels, and plays a fundamental role in ecological systems [1]. It is deposited is preferentially in the bones and teeth of the human body. Some strontium compound such as strontium oxide, strontium hydroxide and strontium carbonate have a strongly irritant effect on skin and mucous membranes especially eyes [2,3]. A radioactive half-life of 29 years makes strontium-90 one of the 
most hazardous products of radioactive fallout [3]. The activity measurement of $\mathrm{Sr}^{2+}$ in aqueous systems is thus of vital importance from an important environmental point of view.

Ionophore-based PVC membrane electrodes are well-established analytical tools routinely used for the measurement of the activity of a wide variety of different ions selectively and directly in complex biological and environmental samples [4-6]. The key ingredient of such plasticized PVC membrane is an incorporated ionophore, which defines the selectivity of the electrodes via selective complex formation with the cation of interest.

Because macrocyclic ligands can form selective and stable complexes with metal ions of compatible dimensions [7], and can potentially be applied to their selective separation and determination [6,8,9], continuous interest has been focused on the design and synthesis of new functionalized macrocycles for specific applications [10]. Several macrocyclic diamides, prepared from salicylaldehade derivatives and appropriate diamino compounds [11], have been reported to have successful use as ion carriers in the construction of PVC-based ion-selective electrodes for $\mathrm{Cu}^{2+}$ [12], $\mathrm{Zn}^{2+}$ [13], $\mathrm{Hg}^{2+}$ [14], $\mathrm{Sr}^{2+}$ [15], $\mathrm{Ca}^{2+}[16], \mathrm{Cs}^{+}[17], \mathrm{Co}^{2+}[18], \mathrm{Ag}^{+}[19]$, and $\mathrm{Be}^{2+}$ ions [20].

In this paper, the suitable cavity size of macrocyclic diamide derivative 1,10-diaza-5,6-benzo-4,7dioxacyclohexadecane-2,9-dione (L) as well as its water insolubility led us to examine its ability as a potential ion carrier in the preparation a new PVC-membrane electrode for $\mathrm{Sr}^{2+}$ ions. It should be noted that, despite urgent need for new potentionmetric sensors for the selective determination of low level amounts of strontium in different samples, there are only limited reports on the $\mathrm{Sr}^{2+}$ ion-selective electrodes in the literature [1,15,21-23].

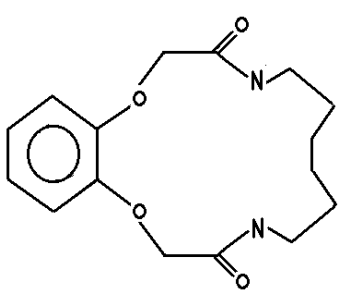

$\mathbf{L}$

\section{Results and Discussion}

Ligands for use as ionophores in a strontium ion-selective electrode should fulfill certain conditions including (1) increased selectivity for $\mathrm{Sr}^{2+}$ over other metal ions, (2) rapid exchange kinetics of the resulting complex and (3) sufficient lipophilicity to prevent leaching of the ligand into the surrounding aqueous solution. The selectivity of ordinary crown ethers for alkaline earth metal ions is much lower than that for alkali cations [6]. However, the introduction of an amide linkage in the polyether ring has been clearly shown to modify the binding properties of the crown compounds in favor of an alkaline earth with respect to alkali metal ions [15,16,20]. Moreover, amide substitution in the cavity of benzocrown ethers may not only contribute to their cation selectivity but also allow the macrocycles to have properties more closely resembling those on the naturally occurring ionophores [26].

To investigate the potential response of the macrocyclic diamide $\mathrm{L}$ for different ions, it was used as a neutral ionophore to prepare PVC-membranes for a wide variety of cations. The potential responses of the most selective electrodes in the concentration range of $10^{-7}-10^{-1} \mathrm{M}$ are shown in Figure 1. As seen, except for the $\mathrm{Sr}^{2+}$ ion-selective electrode, in all other cases the slope of the corresponding 
potential-pM plots is much lower than the expected Nenstian slope of $29.5 \mathrm{mV}$ decade $^{-1}$ for bivalent cations, although over a limited concentration range. As is obvious from the results obtained, $\mathrm{Sr}^{2+}$ ion with the most sensitive response over a wider concentration range seems to be suitably determined with the membrane electrode based on the ligand L. This is due to the highly selective behavior of the ionophore for $\mathrm{Sr}^{2+}$ over other metal ions. The observed selectivity of ionophore $\mathrm{L}$ toward $\mathrm{Sr}^{2+}$ ion is most probably due to the rigid structure of the macrocycle as well as its convenient cavity size (16membered cavity) for the bivalent strontium ion $[7,15]$.

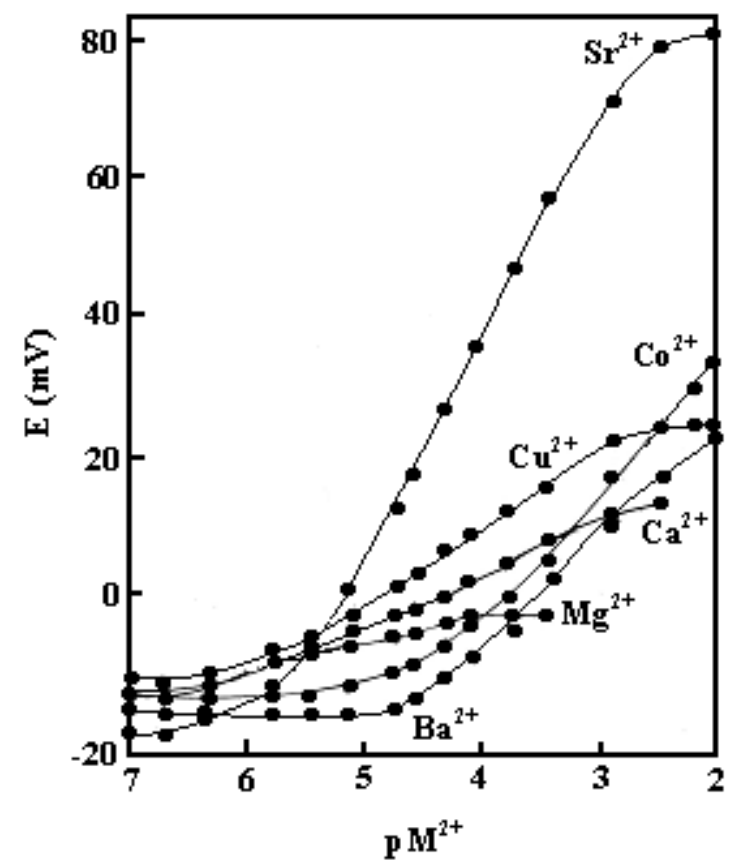

Figure 1. Potential response of various ion-selective membranes based on ionophore L. Conditions: membrane ingredients, $2 \% \mathrm{~L}, 30 \% \mathrm{PVC}, 1.7 \%$ STP and $66.3 \% \mathrm{AP}$, internal solution, $1.0 \times 10^{-3} \mathrm{M}$ of the corresponding cations for each ion-selective sensor.

Some important features of the PVC membranes such as the nature and amount of ionophore, the properties of the plasticizer, the plasticizer/PVC ratio, and specially, the nature of additives used are reported to significantly influence the sensitivity and selectivity of the ion selective electrodes [1220,27]. Thus, the effects of membrane composition, nature and amount of plasticizer, and amount of additive salt on the potential response of the $\mathrm{Sr}^{2+}$ sensor were investigated, and the results are summarized in Table 1.

As with many ionophore-based membrane electrodes, the potential response of the electrode depends on the concentration of lipophilic anionic salts incorporated in the membranes (Nos. 2-4). As seen, addition of STB will increase the sensitivity of the electrode response considerably. Use of $1.7 \%$ STB resulted in a Nernstian behavior (NO.4). The presence of such lipophilic anionic species as tetraphenylborate in cation-selective membrane electrodes is proved to have a beneficial influence on different sensor characteristics. It will not only reduce the ohmic resistance [33] and improve the response behavior and selectivity [27] but also, in cases where the extraction capability of the 
ionophore is poor, enhance the sensitivity of the membrane electrode [28]. Moreover, the additives may catalyze the exchange kinetics at the sample-membrane interface [2].

Table 1. Optimization of membrane ingredients.

\begin{tabular}{|c|c|c|c|c|c|c|}
\hline \multirow{2}{*}{ No } & \multicolumn{4}{|c|}{ Composition (\%) } & \multirow{2}{*}{$\begin{array}{c}\text { Slope } \\
\left(\mathrm{mV} \text { decade }^{-1}\right)\end{array}$} & \multirow{2}{*}{$\begin{array}{c}\text { Linear range }^{\mathrm{a}} \\
(\mathrm{M})\end{array}$} \\
\hline & PVC & Plasticizer & STB & $\mathrm{L}$ & & \\
\hline 1 & 32.5 & $\mathrm{AP}, 66.0$ & 0.5 & 0.0 & 13.5 & $4.6 \times 10^{-5}-1.3 \times 10^{-3}$ \\
\hline 2 & 31.0 & $\mathrm{AP}, 67.0$ & 0.0 & 2.0 & 22.0 & $1.7 \times 10^{-5}-6.3 \times 10^{-3}$ \\
\hline 3 & 31.0 & AP, 66.0 & 1.0 & 2.0 & 25.0 & $3.6 \times 10^{-6}-6.3 \times 10^{-3}$ \\
\hline 4 & 30.0 & AP, 66.3 & 1.7 & 2.0 & 30.5 & $1.6 \times 10^{-6}-3.0 \times 10^{-3}$ \\
\hline 5 & 30.0 & NPOE, 66.3 & 1.7 & 2.0 & 23.5 & $3.6 \times 10^{-6}-1.2 \times 10^{-3}$ \\
\hline 6 & 30.0 & DBP, 66.3 & 1.7 & 2.0 & 16.8 & $3.6 \times 10^{-6}-1.0 \times 10^{-3}$ \\
\hline 7 & 30.0 & DOP, 66.3 & 1.7 & 2.0 & 14.7 & $3.6 \times 10^{-6}-6.3 \times 10^{-3}$ \\
\hline 8 & 30.0 & DMS, 66.3 & 1.7 & 2.0 & 12.8 & $3.6 \times 10^{-6}-6.2 \times 10^{-3}$ \\
\hline
\end{tabular}

${ }^{\mathrm{a}}$ In all cases, the correlation coefficient of the linear calibration plot was at least 0.99 .

Since the nature of plasticizer influences the dielectric constant of the membrane phase, the mobility of the ionophore molecules and the state of ligands [29], it is expected to play an important role in determining the ion-selective characteristics. Thus, five membrane of similar composition (i.e., $30 \%$ PVC, 66.3\% plasticizer, 2\% ionophore and 1.7\% STB) but with five different plasticizers having dielectric constant over the range 4-24, namely DMS $(\varepsilon=4.0)$, DBP $(\varepsilon=6.4)$, DOP $(\varepsilon=7.0)$, NPOE $(\varepsilon$ $=24.0)$ and $\operatorname{AP}(\varepsilon=17.3)$ were prepared and tested (Table 1, Nos 4-8). It is apparent from the performance characteristics that the membrane plasticized with AP had the highest sensitivity with a nice Nernstian slope, widest linear range and lowest detection limit in this series. The slopes of the corresponding potential responses of membranes plasticized with DMS, DBP, DOP and NPOE were $12.8,14.7,16.8$ and $23.5 \mathrm{mV}$ decade $^{-1}$, respectively.

These results indicate that the polar ketone and ether type solvent mediators give a strontium ion sensor with more favorable potentiometric characteristics than nonpolar ester type solvents. This is in agreement with the previous reports showing that the response characteristics and selectivities of multivalent cations of high lipophilicities are favored by the use of high dielectric constant plasticizers [12-20]. As it is obvious from Table 1, the membrane obtained with the PVC/AP/L/STB ratio of $33.0 \% / 66.3 \% / 2.0 \% / 1.7 \%$ (no. 4 ) shows the best response characteristics for selective determination of strontium ion.

Optimum conditioning time for the membrane sensor in a $1.0 \times 10^{-3} \mathrm{M}$ strontium nitrate solution was investigated from 5 to $24 \mathrm{~h}$ and found to be $20 \mathrm{~h}$; it then generates stable potentials when placed in contact with $\mathrm{Sr}^{2+}$ solutions.

The average time required for the $\mathrm{Sr}^{2+}$ ion-selective electrode to reach a potential within $\pm 1 \mathrm{mV}$ of the final equilibrium value was measured after successive immersion of a series of strontium ion solution, each 10-fold different in concentration. The static response time thus obtained was $<15 \mathrm{~s}$ for 
strontium ion concentrations $\leq 10^{-3} \mathrm{M}$, and the potential stayed constant for more than $5 \mathrm{~min}$, after which only a very slow divergence with in the resolution of the $\mathrm{pH}$ meter $( \pm 0.1 \mathrm{mV})$ was recorded.

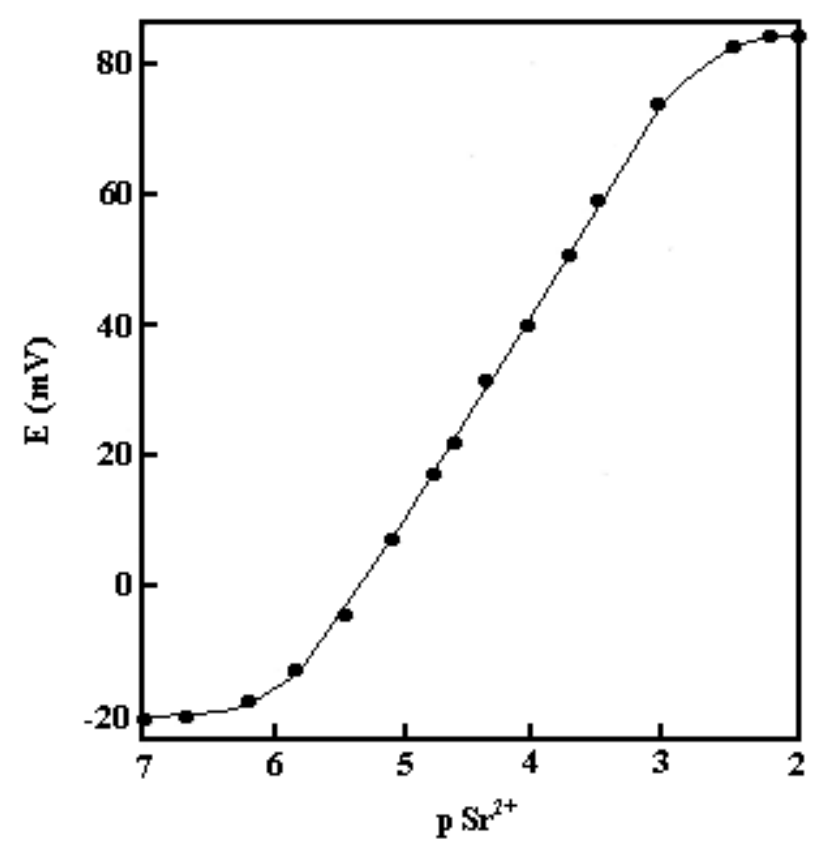

Figure 2. Calibration graph for the strontium ion-selective electrode.

The PVC-membrane electrode based on L prepared under optimized experimental conditions resulted in a potential-pM concentration response with a Nernstian slope of $30.5 \pm 0.6 \mathrm{mV} / \mathrm{decade}$ $(n=7)$ over a wide linear concentration range of $1.6 \times 10^{-6}-3.0 \times 10^{-3} \mathrm{M}$ (Figure 2). The detection limit, as evaluated from the intersection of the two extrapolated segments of the calibration graph, was $6.3 \times 10^{-7} \mathrm{M}$.

The membrane sensor prepared could be used for at least two months without any measurable divergence. After two months, the slope of the calibration curve decreased to $27.0 \mathrm{mV} \mathrm{decade}{ }^{-1}$ without any measurable change in the linear range.

The effect of $\mathrm{pH}$ of test solution on the response of the membrane electrode for a $1.0 \times 10^{-4} \mathrm{M}$ solution of $\mathrm{Sr}^{2+}$ was studied over the $\mathrm{pH}$ range $3.5-11.5$, the results are given in Figure 3 . It is immediately obvious that the potential stays constant from $\mathrm{pH} 4.3$ to 9.4, beyond which sharp increases in potential are observed. The observed sharp change in potential response below $\mathrm{pH} 4.3$ might be due to the simultaneous response of the electrode to $\mathrm{Sr}^{2+}$ ion and $\mathrm{H}^{+}$and at $\mathrm{pH}$ higher than 9.4, it could be because of relatively strong competition between $\mathrm{Na}^{+}$(from $\mathrm{NaOH}$ ) and $\mathrm{Sr}^{2+}$ ions.

The selectivity behavior is obviously one of the most important characteristics of an ion-selective electrode, determining whether a reliable measurement in the electrode proposed can be obtained. Thus, its potential response was investigated in the presence of various interfering foreign cations using the matched potential method (MPM) [30]. The MPM is a recently recommended procedure which gets ride of the limitations of the corresponding methods based on the Nicolski-Eisenman equation for the determination of potentiometric selectivity coefficients. These limitations include non-Nernstian behaviors of interfering ions and inequality of charges of any primary interfering ion. 


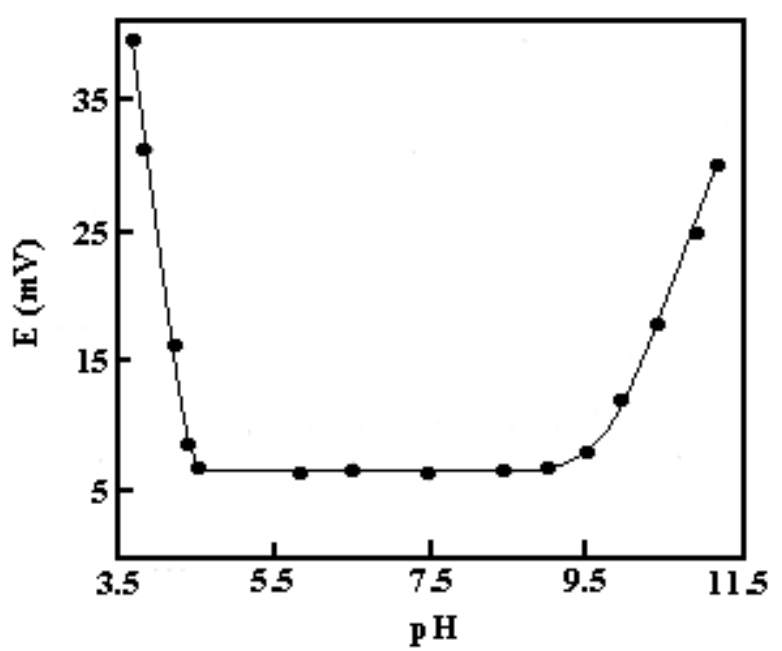

Figure 3. Effect of $\mathrm{pH}$ on the strontium test solution.

According to the MPM, the selectivity coefficient is defined as the activity ratio of the primary ion and the interfering ion that gives the same potential change in a reference solution. Thus, one should measure the change potential upon changing the primary ion activity, and then the interfering ion would be added to an identical reference solution until the same potential change is obtained. The selectivity coefficient is determined as: $K_{A, B}{ }^{M P M}=\Delta a^{\prime}{ }_{A} / a_{B}$, where $\Delta a_{A}=a_{A}{ }^{\prime}-a_{A}, a_{A}$ is the initial primary ion activity and $\mathrm{a}_{\mathrm{A}}{ }^{\prime}$ is the activity of $\mathrm{A}$ in the presence of interfering ion, $\mathrm{B}$, and $\mathrm{a}_{\mathrm{B}}$ is the interfering ion activity. Thus, according to this method, a specific activity (concentration) of the primary ion $\mathrm{A}\left(1.0 \times 10^{-6}-1.0 \times 10^{-5} \mathrm{M} \mathrm{Sr}^{2+}\right)$ is added to a reference solution $\left(5.0 \times 10^{-7} \mathrm{M} \mathrm{Sr}^{2+}\right)$ and the potential is measured. In a separate experiment, the interfering ions $\mathrm{B}$ are successively added to an identical reference solution (from $1.0 \times 10^{-5}$ to $1.0 \times 10^{-3} \mathrm{M}$ of $\mathrm{M}^{\mathrm{n}+}$ ) until the measured potential matched that obtained before by adding the primary ions. The $\mathrm{K}_{\mathrm{A}, \mathrm{B}}{ }^{\mathrm{MPM}}$ value is then calculated as $\Delta \mathrm{a}^{\prime}{ }_{\mathrm{A}} / \mathrm{a}_{\mathrm{B}}$. The resulting values are listed in Table 2. As it is evident from Table 2, most of the interfering ions show low values of selectivity coefficient, indicating negligible interference in the performance of the membrane sensor assembly.

Table 2. Selectivity coefficients of various interfering ions.

\begin{tabular}{cccc}
\hline Interfering ion & $\mathrm{K}_{\mathrm{A}, \mathrm{B}}{ }^{\mathrm{MPM}}$ & Interfering ion & $\mathrm{K}_{\mathrm{A}, \mathrm{B}}{ }^{\mathrm{MPM}}$ \\
\hline $\mathrm{Na}^{+}$ & $1.3 \times 10^{-2}$ & $\mathrm{Ba}^{2+}$ & $9.2 \times 10^{-2}$ \\
$\mathrm{~K}^{+}$ & $3.3 \times 10^{-2}$ & $\mathrm{Co}^{2+}$ & $8 \times 10^{-2}$ \\
$\mathrm{Cs}^{+}$ & $3.3 \times 10^{-2}$ & $\mathrm{Ni}^{2+}$ & $5.4 \times 10^{-2}$ \\
$\mathrm{Rb}^{+}$ & $3.1 \times 10^{-2}$ & $\mathrm{Cu}^{2+}$ & $9.2 \times 10^{-2}$ \\
$\mathrm{Mg}^{2+}$ & $6.3 \times 10^{-2}$ & $\mathrm{~Pb}^{2+}$ & $7.2 \times 10^{-2}$ \\
$\mathrm{Ca}^{2+}$ & $8.9 \times 10^{-3}$ & & \\
\hline
\end{tabular}

In Table 3, the response characteristics of the proposed strontium-sensor are compared with those of the best $\mathrm{Sr}^{2+}$ ion-selective electrodes reported before [2,20,26,27,29]. From the data given in Table 3 it is immediately obvious that the limit of detection of the proposed sensor is superior to those reported 
before, while the other response characteristics and selectivity coefficients obtained in this work are within the same ranges in comparison with those previously reported for the case of the best strontium ion-selective electrodes.

Table 3. Response characteristics and selectivity coefficients for different $\mathrm{PVC}-\mathrm{membrane} \mathrm{Sr}^{2+}$ ionselective electrodes.
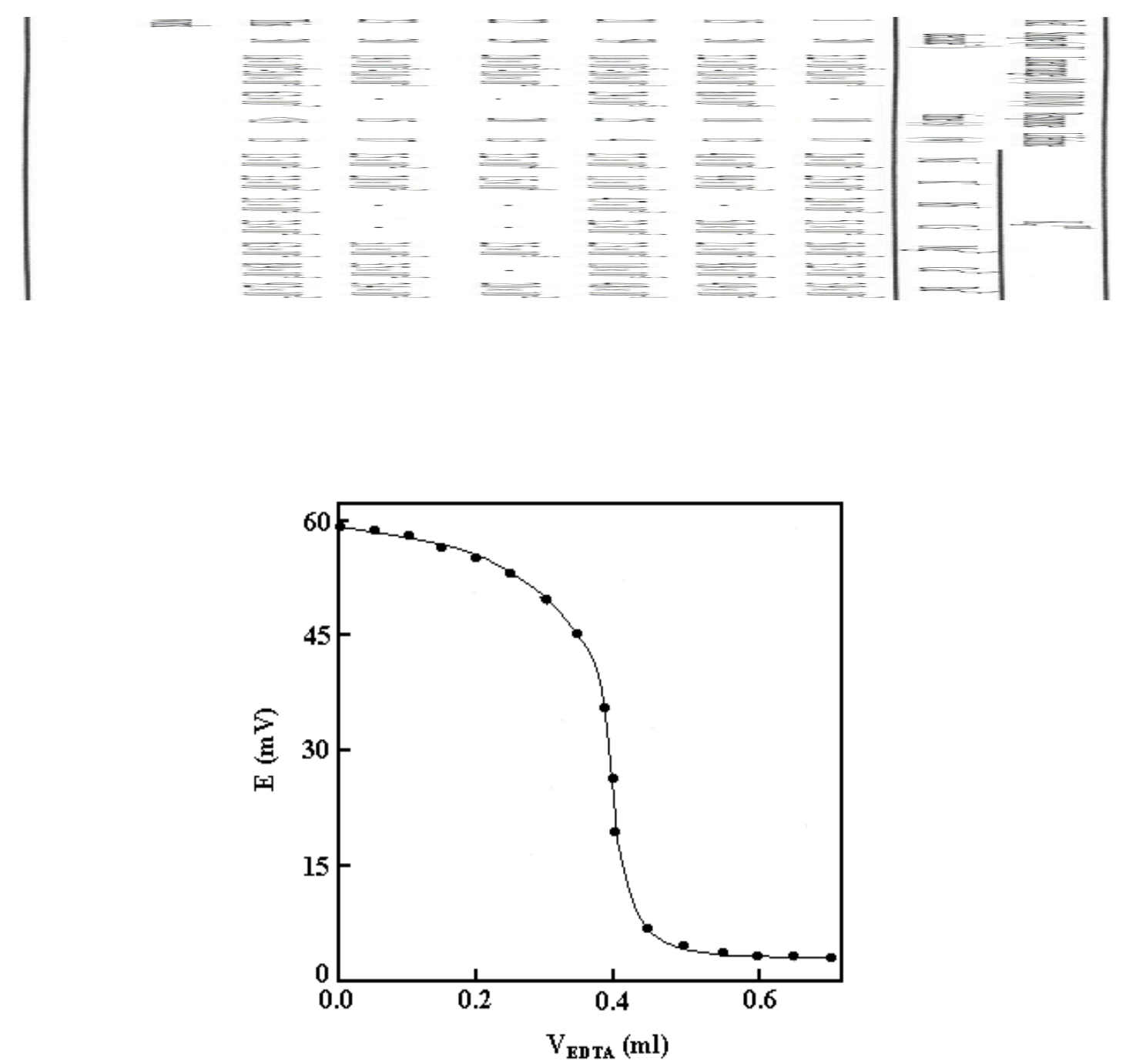

Figure 4. Potentiometric titration curve of $40 \mathrm{ml}$ of $0.001 \mathrm{M} \mathrm{Sr}\left(\mathrm{NO}_{3}\right)_{2}$ with $0.1 \mathrm{M}$ EDTA at pH 6.5, using the proposed sensor as indicator electrode.

The proposed membrane sensor can not only be used for the direct determination of $\mathrm{Sr}^{2+}$ ions in real samples (e.g., quantitative recovery of traces of $\mathrm{Sr}^{2+}$ ion from tap water), but was also found useful as an electrode in potentiometric titration procedures involved strontium ion. As an example, it was successfully applied as an indicator electrode for the titration of $40 \mathrm{ml}$ of $1.0 \times 10^{-3} \mathrm{M}$ concentration of $\mathrm{Sr}^{2+}$ ions, at $\mathrm{pH}$ 5.5, with 0.100 M EDTA solution and results are shown in Figure 4. As seen, the amount of $\mathrm{Sr}^{2+}$ ions in solution can be accurately determined from the resulting titration curve providing a sharp end point. 


\section{Experimental Section}

\subsection{Reagents}

Reagent-grade 2-nitrophenyl octyl ether (NPOE), dibutyl phthalate (DBP), dioctyl phthalate (DOP), acetophenone (AP), dimethylsebacate (DMS), tetrahydrofuran (THF), sodium tetraphenylborate (STB) and high relative molecular weight PVC were purchased from Fluka Chemical Company and used as received. Reagent-grade nitrate salts of all cations used (all from Merck) were of the highest purity available and used without any further purification except for vacuum drying. The macrocyclic diamide L was synthesized and purified in as described elsewhere [24]. Triply distilled and deionized water was used throughout.

\subsection{Electrode Preparation}

The general procedure to prepare the PVC membrane was to mix thoroughly $66.3 \mathrm{mg}$ of plasticizer AP, $30 \mathrm{mg}$ of powdered PVC and $2 \mathrm{mg}$ of ionophore and $1.7 \mathrm{mg}$ of STB in $0.5 \mathrm{ml}$ of methanol and 3.5 $\mathrm{ml}$ of THF. The resulting mixture was transferred into a glass dish of $2-\mathrm{cm}$ diameter. The solvent was evaporated slowly until an oily concentrated mixture was obtained. A Pyrex tube (4-5 mm diameter on the top) was dipped into the oily mixture for about $10 \mathrm{~s}$ so that a non-transparent membrane of about $0.3 \mathrm{~mm}$ thickness was formed. The tube was then pulled out from the mixture and at room temperature for about $1 \mathrm{~h}$. The tube was then filled with internal filling solution $\left(1.0 \times 10^{-3} \mathrm{M}\right.$ strontium nitrate). The electrode was finally conditioned for $20 \mathrm{~h}$ by soaking in a $1.0 \times 10^{-3} \mathrm{M}$ solution of $\operatorname{Sr}\left(\mathrm{NO}_{3}\right)_{2}$. A silver/silver chloride electrode was used as an internal reference electrode.

\subsection{EMF Measurements}

All emf measurements were carried out the following assembly:

$\mathrm{Ag}-\mathrm{AgCl}$ (satd) $|3 \mathrm{M} \mathrm{KCl}|$ internal solution, $1.0 \times 10^{-3} \mathrm{M} \mathrm{Sr}\left(\mathrm{NO}_{3}\right)_{2} \mid \mathrm{PVC}$ membrane $\mid$ test solution | $\mathrm{Ag}-\mathrm{AgCl}, 0.1 \mathrm{M} \mathrm{KCl}$ |

A Metrohm ion analyzer $\mathrm{pH} / \mathrm{mV}$ meter was used for the potential measurements at $25.0 \pm 0.1{ }^{\circ} \mathrm{C}$. Activities were calculated according to Debye-Hückel procedure [25].

\section{Acknowledgements}

The support of this work by a research grant (no. 84123/17) from Iran National Science Foundation (INSF) is gratefully acknowledged.

\section{References and Notes}

1. Jain, A.K.; Gupta V.K.; Raisoni, J.R. Strontium(II)-selective potentiometric sensor based on ester derivative of 4-tert-butylcalix[8]arene in PVC matrix. Sensors 2004, 4,115-124. 
2. Elvers, B.; Hawkins, S.; Russey W. (Eds), Ulmann's Encyclopedia of Industrial Chemistry, $5^{\text {th }}$ Ed., Vol. A25, 1995, VCH Publisher, New York, p. 321.

3. Othmer, K. Encyclopedia of Chemical Technology, $4^{\text {th }}$ Ed., Vol. 22, 1992, John Wiley \& Sons, New York, p. 947.

4. Umezawa Y. (ed.), CRC Handbook of Ion-Selective Electrodes: Selectivity Coefficients, 1990, CRC Press, Boca Raton, FL.

5. Janata, J.; Jasowicz, M.; DeVaney, D.M. Chemical sensors. Anal. Chem., 1998, 70, 179R-208R.

6. Bühlmann, P.; Pretsch, E.; Bakker, E. Carrier based ion-selective electrodes and the bulk optodes. 2. Ionophoresand for potentiometric and optical sensors. Chem. Rev. 1998, 98, 1593-1678.

7. Izatt, R.M.; Pawlak, K.; Bradshaw, J.; Bruening, R.L. Thermodynamic and kinetic data for macrocycle interaction with cations and anions. Chem. Rev. 1991, 91, 1721-2085.

8. Shih, J.-S. Applications of macrocyclic polyethers. J. Chin. Chem. Soc., 1992, 39, 551-559.

9. Lippolis V.; Shamsipur, M. Synthesis, coordination properties, and analytical applicationsof mixed donor macrocycles containing the1,10-phenanthroline sub-unit. J. Iran. Chem. Soc. 2006, 3, 105127.

10. An, H.Y.; Bradshaw, J.S.; Izatt R.M.; Yan, Z. Bis and oligo(benzocrown ethers).Chem. Rev. 1994, 94, 939-991.

11. Sharghi, H.; Eshghi, H. Efficient synthesis of macrocyclic diamides. Tetrahedron 1995, 51, 913922.

12. Shamsipur, M.; Rouhani, S.; Ganjali, M. R.; Eshghi, H.; Sharghi, H. Cu(II)-selective membrane electrode based on a recently synthesized macrocyclic diamide. Microchem. J. 1999, 63, 202-210.

13. Shamsipur, M.; Rouhani, S.; Ganjali, M.R.; Sharghi, H.; Eshghi, H. Zinc-selective membrane potentiometric sensor based on a recently synthesized benzo-substituted macrocyclic diamide. Sens. Actuators B 1999, 59, 30-34.

14. Javanbakht, M.; Ganjali, M.R.; Eshghi, H.; Sharghi, H.; Shamsipur, M. Mercury(II) ion-selective electrode based on dibenzo-diazathia-18-crown-6-dione. Electroanalysis 1999, 11, 81-84.

15. Shamsipur, M.; Rouhani, S.; Sharghi, H.; Ganjali, M.R.; Eshghi, H. Strontium-selective membrane electrode based on some recently synthesized benzo-substituted macrocyclic diamides. Anal. Chem. 1999, 71, 4938-4943.

16. Shamsipur, M.; Khayatian, G.; Kazemi, S.Y.; Niknam, K.; Sharghi, H. The synthesis of 1,4-diaza2,3;8,9-dibenzo-7,10-dioxacyclododecane-5,12-dione and its use in calcium-selective carbon paste electrodes. J. Incl. Phenom. 2001, 40, 303-307.

17. Shamsipur, M.; Kazemi, S.Y.; Sharghi, H.; Niknam, K. Cesium-selective membrane electrode based on a recently synthesized 16-membered macrocyclic diamide. Fresenius J. Anal. Chem. 2001, 371, 1104-1108.

18. Shamsipur, M.; Rouhani, S.; Poursaberi, T.; Ganjali, M.R.; Sharghi, H.; Niknam, K. Cobalt(II)selective coated graphite PVC-membrane electrode based on a recently synthesized dibenzopyridino-substituted macrocyclicdiamide. Electroanalysis 2002, 14, 729.

19. Shamsipur, M.; Kazemi, S.Y.; Niknam, K.; Sharghi, H. A new pVC-membrane electrode based on a thia-substituted macrocyclic diamide in selective potentiometric determination of silver ion. Bull. Korean Chem. Soc. 2002, 23, 53-58. 
20. Shamsipur, M.; Soleymanpour, A.; Akhond, M.; Sharghi, H. New macrocyclic diamides as neutral ionophores for highly selective and sensitive PVC-membrane electrodes for $\mathrm{Be}^{2+}$ ion Electroanalysis 2004, 16, 282-288.

21. Akmal, N.; Zimmer, H.; Mark, H.B. Strontium ion-selective electrode based on a conducting poly(dibenzo-18-crown-6) film. Anal. Lett. 1991, 24, 1431-1443.

22. Qian, G.; Wu, B.B.; Wu, G.; Huang, S.; Yan, Y.; Tian, B. Strontium-selective electrodes based on diamides with pyridine ring as ionophores. Talanta 1998, 47, 1149-1155.

23. Gupta, V.K.; Ludwig, R.; Agarwal, S. Strontium(II) sensor based on a modified calyx[6]arene in PVC matrix. Anal. Sci. 2005, 21, 293-296.

24. Sharghi, H.; Zare, A. Efficient synthesis of some novel macrocyclic diamides using fast addition method. Synthesis 2006, 999-1004.

25. Kamata, S.; Bhale, A.; Fukunaga, Y.; Murata, A. Copper(II)-selective electrode using thiuram disulfate neutral carriers. Anal. Chem. 1988, 60, 2464-2467.

26. Kimura, K.; Kumami, K.; Kitazawa, S.; Shono, T. Calcium-selective polymeric membrane electrodes based on bicyclic poly(ether amide). Anal. Chem. 1984, 56, 2369-2372.

27. Bakker, E.; Bühlmann, P.; Bakker, E. Carrier-based ion selective electrodes and bulk optodes. Part 1. General characteristics. Chem. Rev. 1997, 97, 3083-3112.

28. Eugster, R.; Gehring, P.M.; Morf, W.E.; Spichiger, U.E.; Simon, W. Selectivity-modifying influence of anionic sites in neutral carrier-based membrane electrodes. Anal. Chem. 1991, 63, 2285-2289.

29. Gehrig, P.M.; Morf, W.E.; Welti, M.; Pretsch, E.; Simon, W. Catalysis of ion transfer by tetraphenylborates in neutral carrier-based ion-selective electrodes. Helv. Chim. Acta 1990, 73, 203-212.

30. Umezawa, Y.; Umezawa, K.; Sato, H. Selectivity coefficients for ion-selective electrodes: recommended methods for reporting $\mathrm{K}^{\mathrm{pot}}$ values. Pure Appl. Chem. 1995, 67, 507-518.

(C) 2007 by MDPI (http://www.mdpi.org). Reproduction is permitted for noncommercial purposes. 